\title{
Wie schlimm ist HIV?
}

\author{
Bernard Hirschel ${ }^{a}$, Rachel Spycherb, Maximilien Catsiyannis ${ }^{c}$, Noémie Fracheboud ${ }^{d}$, Christophe Combescure ${ }^{e}$
}

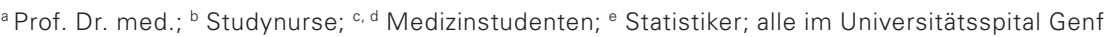

Wer jemanden über seine HIV-Infektion täuscht und so zum ungeschützten Geschlechtsverkehr verleitet und infiziert, macht sich strafbar. Aber wie ist diese Straftat rechtlich zu qualifizieren? Einfache (bis zu drei Jahren Gefängnis oder Busse) oder schwere Körperverletzung (bis zu zehn Jahren Gefängnis)? Ein solcher Fall beschäftigt seit Jahren Schweizer Gerichte.

\section{Der Strafprozess}

Im Jahre 2003 infizierte ein Mann in Zürich, nennen wir ihn A, seinen Freund B mit HIV. Jahre später verklagte B Herrn A, weil dieser ihn über seine Seropositivität getäuscht und ihn so zu ungeschütztem Sex verleitet habe. Das Bezirksgericht verurteilte A zu 33 Monaten Gefängnis, davon 6 Monate unbedingt, aufgrund des Artikels 122 (schwere Körperverletzung) des schweizerischen Strafgesetzbuches. Dazu kamen Kosten für das Gerichtsverfahren, Schadenersatz und Genugtuung, die zusammen wohl 100000 Franken erreichen.

A appellierte ans Zürcher Obergericht, das das Urteil bestätigte [1]. Im Jahre 2013 entschied das Bundesgericht in Lausanne [2]: Das Zürcher Obergericht solle durch ein Gutachten die Schwere der HIV-Infektion unter Berücksichtigung der therapeutischen Fortschritte beurteilen lassen und dann neu entscheiden, ob der Tatbestand die Anwendung des Artikels 122, oder aber des Artikels 123 rechtfertige.

Schweizerisches Strafgesetzbuch Art. 122; schwere Körperverletzung

Wer vorsätzlich einen Menschen lebensgefährlich verletzt, wer vorsätzlich den Körper, ein wichtiges Organ oder Glied eines Menschen verstümmelt oder ein wichtiges Organ oder Glied unbrauchbar macht, einen Menschen bleibend arbeitsunfähig, gebrechlich oder geisteskrank macht, das Gesicht eines Menschen arg und bleibend entstellt, wer vorsätzlich eine andere schwere Schädigung des Körpers oder der körperlichen oder geistigen Gesundheit eines Menschen verursacht, wird mit Freiheitsstrafe bis zu zehn Jahren oder Geldstrafe nicht unter 180 Tagessätzen bestraft.

Art. 123; einfache Körperverletzung

Wer vorsätzlich einen Menschen in anderer Weise an Körper oder Gesundheit schädigt, wird, auf Antrag, mit Freiheitsstrafe bis zu drei Jahren oder Geldstrafe bestraft. In leichten Fällen kann der Richter die Strafe mildern.

\section{Die Schwere der HIV-Infektion}

\section{Die Einführung von HAART}

Ohne wirksame Behandlung sind 12 Jahre nach der HIV-Infektion etwa die Hälfte der Patienten verstorben [3]. Ab etwa 1996 wurde aber der natürliche Verlauf durch die Verfügbarkeit von wirksamen Medikamenten völlig verändert. Die Abbildung 1 zeigt die Inzidenz der opportunistischen Infektionen und Tumoren (AIDS) vor und nach Beginn der hochaktiven anti-retroviralen Therapie (HAART) bei Patienten der schweizerischen HIV-Kohortenstudie [4]:

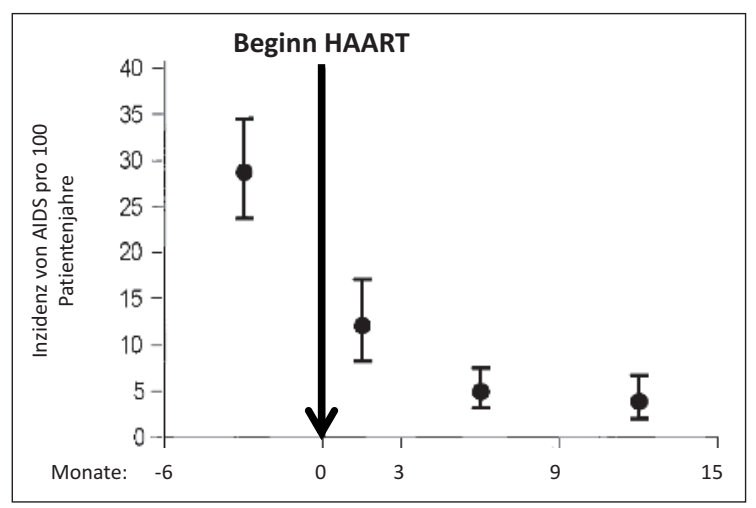

Abbildung 1: Inzidenz der opportunistischen Infektionen und Tumoren (AIDS) vor und nach Beginn der hochaktiven antiretroviralen Therapie (HAART) bei Patienten der schweizerischen HIV-Kohortenstudie.

Allerdings war HAART keine einfache Behandlung. 1996 war die Kombination von Indinavir, Zidovudin und 3-TC marktführend: Patienten nahmen um $6 \mathrm{Uhr}$ morgens auf nüchternen Magen zwei Tabletten Indinavir, zum Frühstück um 8 Uhr eine Tablette Zidovudin und eine Tablette Lamivudin, um 14 Uhr wiederum lndinavir, zum Nachtessen um 18 Uhr Zidovudin und 
Lamivudin, und um 22 Uhr lndinavir [5]. Nebenwirkungen (Durchfall, Übelkeit, Blutarmut, Nierensteine, eingewachsene Zehennägel, Veränderung des Aussehens mit Fettanlagerung im Bauchbereich und Fettschwund im Gesicht - die sogenannte Lipodystrophie) waren häufig.

\section{Weitere Fortschritte 1996-2015}

Neuere Medikamente, wie z.B. Efavirenz, Tenofovir und Lopinavir mussten nur noch einmal oder zweimal täglich verabreicht werden. Nebenwirkungen, insbesondere die Lipodystrophie, wurden seltener. Dies erleichterte die Einhaltung der Behandlungsvorschriften, was wiederum den Therapieerfolg verbesserte $[6,7]$. Auch das Resistenzproblem wurde weitgehend gelöst. Drei Substanzen - Etravirin, Raltegravir und Darunavir - brachten Besserung. In Kombination lösen sie auch die schwersten Resistenzprobleme. Wegen Resistenz nicht-behandelbare Patienten gibt es praktisch nicht mehr [8].

Eine weitere Verbesserung war die Kombination von verschiedenen aktiven Substanzen in einer Pille. Die Kombinationspräparate Atripla ${ }^{\circledR}$, Eviplera ${ }^{\circledR}$, Stribild ${ }^{\circledR}$ und Triumeq ${ }^{\circledast}$ erlauben heute in der Schweiz die HIVBehandlung mit einer Pille pro Tag.

\section{Überleben mit HIV - einst und jetzt}

Bei der Beurteilung der Folgen einer HIV-Infektion spielt die Frage nach der Lebenserwartung eine wichtige Rolle. Wie erwähnt, waren ohne Behandlung zwölf Jahre nach der Infektion etwa die Hälfte der Infizierten tot: Bei einem Durchschnittsalter der Betroffenen von etwa 30 Jahren eine ganz massive Lebensverkürzung. Wie sieht dies heute aus, unter Berücksichtigung der wirksamen Behandlung? Die Frage ist nicht ganz einfach zu beantworten:
- Die Beobachtungszeit ist noch kurz. Eine Überlebenskurve ist erst dann ganz genau, wenn alle Beteiligten verstorben sind. Bei der relativ jungen HIV-Bevölkerung müsste man mehr als 50 Jahre warten!

- Wirksame Medikamente gibt es seit weniger als 20 Jahren. Späte, schwere Nebenwirkungen oder Wirkungsverlust sind zwar unwahrscheinlich, würden aber das Überleben beeinflussen, ohne dass man das heute feststellen könnte.

- Die HIV-Behandlung wurde auch in den letzten Jahren noch verbessert. Dies ist aber schwer nachzuweisen, weil bei spätem Behandlungsbeginn die Beobachtungszeit fehlt.

- Auch bei kurzer Beobachtungszeit ist es möglich, das Überleben von HIV-Infizierten mit dem einer HIVnegativen Kontrollgruppe, z.B. der Schweizer Bevölkerung gleichen Alters, zu vergleichen. Ist die Sterblichkeit gleich, extrapoliert man in die Zukunft und erwartet eine ähnliche Lebenserwartung. Hier gilt es zu berücksichtigen, dass sich HIV-Positive und HIVNegative nicht nur bezüglich der HIV-Infektion unterscheiden. HIV-Positive rauchen z.B. häufiger als HIV-Negative, was die Lebenserwartung negativ beeinflusst. Viele haben sich neben dem HIV auch mit Hepatitis-Viren infiziert, was nach 20 und mehr Jahren zu tödlichem Leberversagen oder Krebs führen kann. In der Schweiz waren 2009 nur 9\% der Todesfälle bei HIV-Positiven auf Aids zurückzuführen [9]!

\section{Prognose der HIV-Infektion: Daten aus der Schweizer HIV-Kohortenstudie (SHCS) [10]}

Eine im Jahre 2003 erschienene Arbeit untersuchte die Sterblichkeit in der SHCS zwischen 1997 und 2001. Die HIV-positive Population wurde in verschiedene Kategorien aufgeteilt und die Sterblichkeit mit derjenigen

Tabelle 1: Sterblichkeit von HIV-Patienten unter erfolgreicher HAART, 1997-2001.

\begin{tabular}{llll}
\hline Kategorie & $\begin{array}{l}\text { Sterblichkeit der HIV-Patienten } \\
\text { pro } 1000 \text { Beobachtungsjahre }\end{array}$ & $\begin{array}{l}\text { Referenzwert: Sterblichkeit von } \\
\text { vergleichbaren HIV-negativen } \\
\text { Schweizern }\end{array}$ & Übersterblichkeit \\
\hline $\mathrm{HCV}-, \mathrm{HIV}+$ & 7,8 & 3,6 & 4,2 \\
\hline $\mathrm{HCV}+, \mathrm{HIV}+$ & 23,4 & $1,7^{*}$ & 21,7 \\
\hline
\end{tabular}

* Hepatitis C und HIV koexistieren vor allem in Drogenabhängigen. Diese sind im Durchschnitt jünger als die nur mit HIV Infizierten; deshalb der niedrigere Referenzwert für die Mortalität.

Tabelle 2: Sterblichkeit von HIV-Patienten ohne, oder unter nicht erfolgreicher HAART, 1997-2001.

\begin{tabular}{llll}
\hline Kategorie & $\begin{array}{l}\text { Sterblichkeit der HIV-Patienten } \\
\text { pro } \mathbf{1 0 0 0} \text { Beobachtungsjahre }\end{array}$ & Referenzwert & Übersterblichkeit \\
\hline $\mathrm{HCV}-, \mathrm{HIV}_{+}$ & 123 & 5,6 & 117,4 \\
\hline $\mathrm{HCV}+, \mathrm{HIV}+$ & 115 & 2,3 & 112,7 \\
\hline
\end{tabular}


Tabelle 3: Fragebogen der Umfrage.

Bitte vergleichen Sie die folgenden Krankheiten mit der HIV-Infektion.

1. Einen hohen Blutdruck zu haben ist
$\square$ schlimmer
$\square$ gleich schlimm
weniger schlimm (als eine HIV-Infektion)

2. Eine Verletzung, die eine schlimme Narbe im Gesicht zurücklässt, ist

$\square$ schlimmer $\quad \square$ gleich schlimm $\quad \square$ weniger schlimm (als eine HIV-Infektion)

3. Diabetes (Zuckerkrankheit), mit Tabletten behandelt, ist

$\square$ schlimmer $\quad \square$ gleich schlimm $\quad \square$ weniger schlimm (als eine HIV-Infektion)

4. An Arthrose zu leiden und deswegen am Stock zu gehen ist
$\square$ schlimmer
$\square$ gleich schlimm
weniger schlimm (als eine HIV-Infektion)

5. Diabetes (Zuckerkrankheit), mit täglichen Insulinspritzen behandelt, ist

$\square$ schlimmer $\quad \square$ gleich schlimm $\quad \square \quad$ weniger schlimm (als eine HIV-Infektion)

6. Ein Auge zu verlieren ist

$\square$ schlimmer $\quad \square$ gleich schlimm $\quad \square$ weniger schlimm (als eine HIV-Infektion)

7. Einen Krebs zu haben, der operiert werden muss (wie z.B. Brustkrebs bei einer Frau oder Prostatakrebs bei einem Mann), ist $\square$ schlimmer $\quad \square$ gleich schlimm $\quad \square$ weniger schlimm (als eine HIV-Infektion)

8. Hirnschlag und als Folge eine einseitige Lähmung an Arm und Bein ist $\square$ schlimmer $\quad \square$ gleich schlimm $\quad \square$ weniger schlimm (als eine HIV-Infektion)

9. $\quad$ Blind zu werden ist

$\square$ schlimmer $\quad \square$ gleich schlimm

$\square$

10. Krebs ohne Aussichten auf Heilung ist weniger schlimm (als eine HIV-Infektion)

$\square$ schlimmer $\quad \square$ gleich schlimm

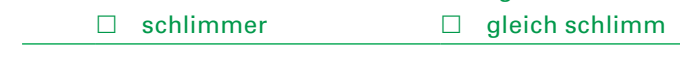

der Schweizer Allgemeinbevölkerung gleichen Alters verglichen (Referenzwert). Die Daten zeigen den überwiegenden Einfluss der Hepatitis-C-Infektion auf die Sterblichkeit bei erfolgreich mit HAART behandelten Patienten (Tab. 1) und andrerseits die Wirkung der Behandlung, mit Reduktion der Sterblichkeit um mehr als 90\% (von 123 pro Tausend Patientenjahre auf 7,8, vgl. Tab. 2 mit Tab. 1).

Die Mortalität ist seither weiter gesunken [11]; Behandlungsbeginn nach 2001 ist prognostisch günstiger als Behandlungsbeginn 1996 bis 2000 (op. cit. Tab. 2). Es besteht aber immer noch ein erheblicher Unterschied zwischen der Mortalität der HIV-Positiven und derjenigen der typischen Kunden von LebensversicherungsGesellschaften (die wiederum niedriger ist als die Mortalität der Allgemeinbevölkerung).

\section{Lebensqualität, Nebenwirkungen der Medika- mente, "frühes Altern», Fortpflanzung}

HAART bedeutet die lebenslange Einnahme von Medikamenten. Viele Patienten wären bereit, für die definitive Heilung von HIV erhebliche Risiken einzugehen. Leider gibt es gegenwärtig keine Hoffnung, dass dies in absehbarer Zeit möglich sein wird. Eine ärztliche Betreuung mit regelmässigen Kontrollen alle drei bis sechs Monate ist erforderlich.

Nebenwirkungen waren beim ersten Einsatz von HAART fast unvermeidlich. Dies hat sich zwar seither verbessert; mit mehr als 20 Medikamenten zur Auswahl ist es heute fast immer möglich, eine verträgliche
Kombination zu finden. Befürchtungen, dass die Behandlung langfristig das Auftreten von Herz- und Kreislaufkrankheiten fördere und, zusammen mit der HIV-Infektion selber, den Altersprozess beschleunige, haben sich bisher nicht bewahrheitet.

Diskriminierung, oder zumindest die Angst davor, lastet schwer auf vielen HIV-Infizierten. Dies hat praktische Auswirkungen, etwa die Schwierigkeit, eine Lebensversicherung abzuschliessen, was wiederum die Kreditwürdigkeit herabsetzt. Andere trauen sich nicht, eine neue Stelle anzunehmen in der Angst, ihre Seropositivität und sexuelle Orientierung würde via Pensionskasse im Betrieb bekannt.

Die Fortpflanzung betreffend sind Befürchtungen zwar objektiv unbegründet - Übertragung von HIV von einer mit HAART behandelten Mutter auf das Kind ist praktisch unmöglich, wie auch Übertragung von einem erfolgreich Behandelten durch Geschlechtsverkehr. Trotzdem bleibt Offenheit bezüglich HIV unter Sexualpartnern ein Thema. Viele berichten, sie seien von ihren Partnern wegen HIV verlassen worden und andere getrauen sich gar nicht mehr, eine neue Beziehung einzugehen.

\section{Was denken die Leute? Eine Umfrage}

In einem demokratischen Staat ist die Einschätzung einer Straftat wenigstens zum Teil Ausdruck der öffentlichen Meinung. Es ist deswegen von Interesse, was der Bürger denkt: HIV, eine schwere oder eine einfache 
Tabelle 4: Ergebnis der Befragung.

\begin{tabular}{|c|c|c|c|}
\hline Befragte Bevölkerung & $\mathbf{N}$ & Score & $\mathbf{P}$ \\
\hline Jus-Studenten & 185 & 8,1 & \multirow{2}{*}{$\begin{array}{l}-\quad<10^{-3} \text { (t-Test) zwischen Jus- und Medizin- } \\
\text { studenten } \\
\text { - Nicht signifikant zwischen Medizinstuden- } \\
\text { ten und Spitalangestellten }\end{array}$} \\
\hline Medizin-Studenten & 217 & 6,9 & \\
\hline Alle HIV-negativen befragten Personen & 737 & 7,2 & \multirow{2}{*}{$\begin{array}{l}-<10^{-3} \text { (t-Test) zwischen HIV-Positiven und } \\
\text { HIV-Negativen }\end{array}$} \\
\hline HIV-positive Patienten & 297 & 4,8 & \\
\hline
\end{tabular}

Körperverletzung? Wie reflektieren sich die therapeutischen Fortschritte in den Ansichten einer breiten Öffentlichkeit?

Um dies herauszufinden, haben wir im Jahre 2014 eine Umfrage veranstaltet mit dem Ziel, die Schwere der HIV-Infektion mit derjenigen anderer Krankheiten zu vergleichen (Tab. 3).

Dieser Fragebogen wurde 2014 von insgesamt 1043 Personen ausgefüllt, davon waren 297 HIV-positive Patienten in Genf und im benachbarten Annecy. 737 waren HIV-negativ: Je etwa 200 Jus- und Medizinstudenten im zweiten Studienjahr, 236 Spitalangestellte (vorwiegend Ärzte und Krankenschwestern) und 99 andere. Der Auswertung lag folgende Überlegung zugrunde:

- Wer HIV als schlimm einstuft, wird von vielen der angeführten Krankheiten meinen, sie seien weniger oder gleich schlimm als HIV.

- Umgekehrt derjenige, der HIV als relativ harmlos einstuft: Kaum eine der angeführten Krankheiten erscheinen ihm weniger oder gleich schlimm.
Jeder Fragebogen bekam folglich einen Score entsprechend der Anzahl der angekreuzten «weniger schlimm»- oder "gleich schlimm»-Antworten. Die Resultate sind in der Tabelle 4 zusammengefasst.

Je nach der befragten Bevölkerungsgruppe sind also die Antworten verschieden. Während HIV-Positive finden, die HIV-Infektion sei etwa so schlimm wie eine Arthrose oder Diabetes, denken Jus-Studenten eher an Hirnschlag mit Hemiplegie. Die Abbildung 2 illustriert den Kontrast zwischen den beiden befragten Gruppen besonders deutlich.

Woher kommen diese Unterschiede? Zwei hauptsächliche Erklärungen bieten sich an:

- Die neuen Behandlungen haben die Prognose der HIV-Infektion von Grund auf verändert. Patienten erleben diesen Fortschritt am eigenen Körper, Medizinstudenten und anderes medizinisches Personal kennen ihn wenigstens teilweise aus Patientenkontakten und Unterricht. Das Bild aber, das sich die weitere Öffentlichkeit von HIV und AIDS macht,

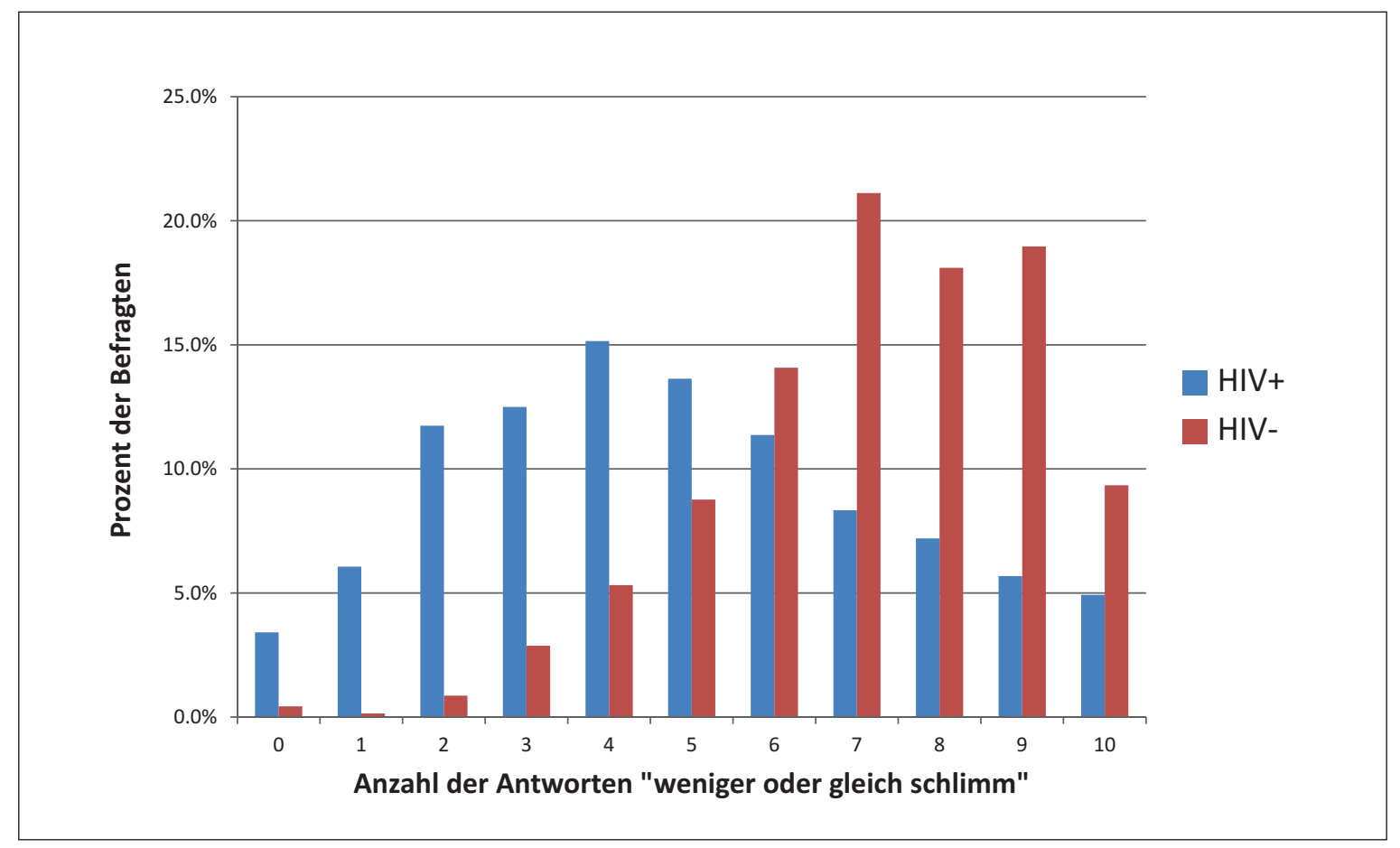

Abbildung 2: Vergleich der Antworten der Seropositiven und der Seronegativen. 
hinkt diesem Fortschritt nach; dies mag den Unterschied zwischen Medizin- und Jus-Studenten erklären.

- Menschen mit schweren Krankheiten oder Verletzungen beurteilen diese als weniger schlimm als Nicht-Kranke und Nicht-Verletzte, nach dem Prinzip: «Man gewöhnt sich an alles» [12].

\section{Der Gerichtsentscheid}

Wie hätten Sie, liebe Leserin, lieber Leser, entschieden? Ist A nach Artikel 122 (schwere Körperverletzung) oder 123 (einfache Körperverletzung) zu verurteilen? Die zweite Strafkammer des Zürcher Obergerichts hat am 29.6.2015 ihren Entscheid gefällt [13]. Sie bestätigte dabei die Qualifizierung der Tat als schwere Körperverletzung.

Wenn das Obergericht im Hauptpunkt auch das vorherige Urteil bestätigte, so revidierte es doch das Strafmass, denn seit der Straftat waren mehr als 10 Jahre vergangen, während derer sich der Beschuldigte nichts Weiteres hat zu Schulden kommen lassen und sogar beruflich in der HIV-Prävention tätig wurde. Die Dauer der Freiheitsstrafe verminderte sich von 30 auf 24 Monate, was den bedingten Vollzug ermöglicht, und die Genugtuungssumme von 50000 Franken auf 35000 Franken.

Der Angeklagte hat aber gegen diesen Entscheid Beschwerde beim Bundesgericht erhoben - es folgt also noch eine Fortsetzung. Die Chancen dieser Beschwerde stehen wohl nicht sehr gut, hat doch das BG im Frühjahr 2015 die Qualifizierung der HIV-Infektion als schwere Körperverletzung in einem andern Fall - dem «Heiler von Bern» - ausdrücklich bestätigt [14].

Korrespondenz:

Prof. Dr. med. B. Hirschel

13 , chemin des Lucioles

CH-1234 Vessy

Tel. 0227842695

bernard.hirschel[at]

hcvge.ch

\section{Danksagung}

Dank an Frau Susanne Polydor, die die juristischen Aspekte des Manuskripts kontrolliert hat, an die Doktores J.-P. Bru und Jacques Gillet für die Befragung ihrer Patienten in Annecy, und an Prof. Sylvain Marchand für die Befragung der Jus-Studenten.
Literatur

1 www.gerichte-zh.ch/fileadmin/user_upload/entscheide/ oeffentlich/SB110505-O1.pdf

2 www.bger.ch/index/juridiction/jurisdiction-inherit-template/ jurisdiction-recht/jurisdiction-recht-leitentscheide1954.htm, Urteil 139 IV 214

3 Koblin BA, van Benthem BH, Buchbinder SP, Ren L, Vittinghoff E, Stevens CE et al. Long-Term Survival after Infection with Human Immunodeficiency Virus Type 1 (HIV-1) among Homosexual Men in Hepatitis B Vaccine Trial Cohorts in Amsterdam, New York City, and San Francisco, 1978-1995. American Journal of Epidemiology. 1999;150:1026-30.

4 Ledergerber B, Egger M, Erard V, Weber R, Hirschel B, Furrer H, et al. AIDS-Related opportunistic illnesses occurring after initiation of patent antiretroviral therapy. The Swiss HIV Cohort Study. JAMA. 1999;282(23):2220-6.

5 Hammer SM, Squires KE, Hughes MD, Grimes JM, Demeter LM, Currier JS et al. A controlled trial of two nucleoside analogues plus indinavir in persons with human immunodeficiency virus infection and CD4 cell counts of 200 per cubic millimeter or less. N Engl J Med. 1997; 337(11):725-33.

6 Staszewski S, Morales-Ramirez J, Tashima KT, Rachlis A, Skiest D, Stanford J et al. Efavirenz plus zidovudine and lamivudine, efavirenz plus indinavir, and indinavir plus zidovudine and lamivudine in the treatment of HIV-1 infection in adults. Study 006 Team. N Engl J Med. 1999 Dec 16;341(25\}:1865-73.

7 Sax PE, Tierney C, Collier AC, Fischl MA, Mollan K, Peeples L et al. Abacavir-lamivudine versus tenofovir-emtricitabine for initial HIV-1 therapy. N Engl J Med. 2009;361 (23):2230-40.

8 Fagard C, Colin C, Charpentier C, Rami A, Jacomet C, Yeni P et al. Long-term efficacy and safety of raltegravir, etravirine, and darunavir/ritonavir in treatment-experienced patients: week 96 results from the ANRS 139 TRIO trial. J Acquir Immune Defie Syndr 2012;59(5):489-93.

9 Weber R, Ruppik M, Rickenbach M, Spoerri A, Furrer H, Battegay M et al. Decreasing mortality and changing patterns of causes of death in the Swiss HIV Cohort Study. HIV Medicine. 2013;14:195.

10 Jaggy C, von Overbeck J, Ledergerber B, Schwarz C, Egger M, Rickenbach M et al. Mortality in the Swiss HIV Cohort Study (SHCS) and in the Swiss general population. Lancet. 2003;362:877-9.

11 Kaulich-Bartz J,Dam W, May MT, Ledergerber B, Widmer U, Phillips AN, Grabar S et al. Insurability of HIV-positive people treated with antiretroviral therapy inEurope: collaborative analysis of HIV cohort studies. AlOS. 2013;27:1641-55.

12 Kahnemann D. Thinking fast and slow. Penguin Books, Penguin Random House UK; 2012. p 404-6.

13 Obergericht des Kantons Zürich, II. Strafkammer, Urteil vom 29.6.2015, SB130132.

14 www.bger.ch/index/juridiction/jurisdiction-inherit-template/ jurisdiction-recht/jurisdiction-recht-leitentscheide1954.htm, BGE 141 IV 97. 